La constitution des autorités numériques dans la production et la circulation de l'information

\title{
Assogba Henri (dir.) : Journalismes spécialisés à l'ère numérique
}

Marie-Ève Saint Georges

\section{(2) OpenEdition}

\section{Journals}

Édition électronique

URL : https://journals.openedition.org/edc/11063

DOI : $10.4000 /$ edc. 11063

ISSN : 2101-0366

Éditeur

Université de Lille

Édition imprimée

Date de publication : 1 décembre 2020

Pagination : 199-202

ISBN : ISBN : 978-2-917562-24-6

ISSN : 1270-6841

Référence électronique

Marie-Ėve Saint Georges, « Assogba Henri (dir.) : Journalismes spécialisés à l'ère numérique », Études de communication [En ligne], 55 | 2020, mis en ligne le 01 décembre 2020, consulté le 03 janvier 2023. URL : http://journals.openedition.org/edc/11063 ; DOI : https://doi.org/10.4000/edc.11063

Ce document a été généré automatiquement le 3 janvier 2023.

Tous droits réservés 


\title{
Assogba Henri (dir.) : Journalismes spécialisés à l'ère numérique
}

\author{
Marie-Ève Saint Georges
}

\section{RÉFÉRENCE}

Assogba H. (dir.) (2020). Journalismes spécialisés à l'ère numérique, Québec, Presses de l'Université Laval.

1 Publié par les Presses de l'Université Laval début 2020, les enjeux liés aux spécialisations en journalisme à l'ère numérique - tels qu'ils sont présentés dans la partie introductive - sont ancrés à la fois dans la formation et la pratique de jeunes journalistes. À travers les douze chapitres proposés, nous nous saisissons directement de l'émergence de problématiques qui touchent les néo-journalistes professionnels, dans un contexte complexifié par l'impact difficile à juguler sur le plan économique et où les rédactions sont désormais plurimédias. Ces problématiques se déclinent suivant la phrase d'accroche, dans le propos introductif, qui invite à penser «Le journalisme [comme étant] à la fois un et multiple »: une profession à la fois rêvée par les prétendants à ce métier en pleine mutation et particulièrement décriée voire défiée. Il s'agit alors de cerner les contours de cette ambivalence du journalisme, entre le travail réalisé par des généralistes et celui de journalistes aujourd'hui « hyperspécialisés » sur le web. Comme le mentionne d'emblée le directeur de l'ouvrage, Henri Assogba, ce livre résulte d'un colloque du réseau Théophraste, soit le regard croisé d'une quinzaine d'enseignants-chercheurs rattachés à des formations en journalisme dans des zones francophones. C'est également là que réside tout l'intérêt de contributions en provenance de l'Europe, mais aussi du Québec ou encore du Burkina-Faso.

2 La logique qui prévaut, afin de saisir aussi la vitesse comme vecteur d'accélérateur de ce journalisme spécialisé, consiste à décliner cette réflexion sur des formes de spécialisations suivant trois grands axes. 
3 D'abord, l'axe portant sur les enjeux liés aux spécialisations fonctionnelles met en évidence le web journalisme avec l'usage des réseaux sociaux comme vecteur de valorisation. L'étude d'Amandine Degand présente ainsi des «journalistes stagiaires prêts à l'emploi ». De son côté, Rayya Roumanos compare des pratiques sur le journalisme de données en pointant les paradoxes d'une hyperspécialisation en butte à des effets indésirables sur le plan éditorial.

4 Ensuite, l'axe qui a trait aux enjeux liés aux spécialisations thématiques met en exergue une réalité du journalisme au Burkina-Faso. L'on y perçoit, dès l'intitulé de la recherche, une double entrée possible dans l'identité de «journalistes de rang ou journalistes touche-à-tout » (p. 147). Si être un journaliste spécialisé dans la couverture d'événements sportifs, par exemple, rejoint la réalité de beaucoup de rédactions dans le monde avec des journalistes spécialisés, l'Afrique subsaharienne - et le cas burkinabé en particulier - n'offre pas cette possibilité. Lassané Yaméogo interroge cette quasiabsence de journalistes spécialisés, à travers « Les dynamiques sociales ("du dedans" et "du dehors") qui entravent l'émergence d'un journalisme spécialisé au Burkina-Faso » (p.148), dans un contexte marqué par des attaques terroristes très fréquentes ces dernières années.

5 Aux confins de la spécialisation thématique et de la spécialisation géographique, l'une des contributions de cet ouvrage signée par Paula de Sauza Pes s'appuie notamment sur sa thèse de doctorat réalisée à l'Université Grenoble Alpes en 2014. La résonance avec les problématiques liées à l'immigration en cette année 2020 est d'autant plus forte. Le travail journalistique se retrouve encore plus complexifié. Les journalistes sont décriés, défiés dans les banlieues, les reportages ne sont pas plus aisés à produire pour le web. Partant du postulat sur le manque d'études sur les journalistes spécialisés dans la couverture de l'immigration (Bonnafous, 1999'1), considérant aussi que ce thème demeure souvent un sous-champ dans l'actualité traitée par les services « Société » des médias, la recherche montre une plus forte propension à la couverture de ces sujets dans la presse quotidienne nationale $(\mathrm{PQN})$ que dans la presse quotidienne régionale $(\mathrm{PQR})$. Cela présupposerait la disponibilité de journalistes du Monde, par exemple, à montrer des situations d'urgence ou à bénéficier d'un temps nécessaire pour une enquête au long cours. Quant au numérique, si la question de savoir comment l'accélération ainsi que la profusion des canaux possibles pour publier des sujets portant sur l'immigration demeure ouverte, le constat d'une thématique « de plus en plus visible à mesure que des enjeux politiques deviennent plus prégnants » (p. 186) s'affirme largement ces quarante dernières années.

6 À travers cette problématique sur un journalisme spécialisé, l'ensemble des chapitres présente une unité dans les mutations liées au numérique. Mutations qui impactent toujours plus l'identité et le métier de journaliste. Les études se situent dans le droit prolongement des recherches (Neuveu, Rieffel, Ruellan, 2002²) en la matière ou encore de l'évolution qui s'opèrent dans les rédactions (Charon, 1993 et 201633).

7 La dernière partie de l'ouvrage est consacrée à l'axe sur les spécialisations géographiques. Ce sont successivement une étude menée par l'UCL et dirigée par Lara Van Dievoet sur la question de la proximité dans le traitement de l'actualité locale en Belgique francophone, et les travaux de l'équipe de recherche, sous la direction de Marie-Ève Carignan, concernant la porosité entre publicité native et marketing de contenu au sein de rédactions en mouvement permanent. Si la plus ou moins grande proximité avec un lectorat reste une règle journalistique à part entière, la question 
posée par une forme d'emprise du marketing directement sur le territoire journalistique correspond beaucoup plus à la quête d'un nouveau modèle économique dans des rédactions bousculées par le numérique.

8 L'épilogue est signé par Thomas Kent, sur « La propagation des fausses nouvelles dans les conflits entre pays[...] beaucoup plus efficace, plus efficace même qu'au $\mathrm{XX}^{\mathrm{e}}$ siècle» (p. 241-244). Son propos apporte à la réflexion générale de ce livre, l'œil expérimenté de l'enseignant de l'université américaine de Columbia. Il boucle ainsi un questionnement sur la spécialisation des journalistes autant que sur leurs façonnages au sein des structures de formation en journalisme. Au final, l'inévitable triptyque entre "Propagande, vérité et avenir de l'information» (également sujet de la conférence inaugurale de l'ancien journaliste d'Associated Press, lors de ce colloque du réseau Théophraste) signifie pour Thomas Kent que «[La] nouvelle spécialité journalistique dont nous avons besoin ne s'occupe pas seulement de la vérification des faits, mais de l'analyse et du démasquage de toute la toile de la désinformation » (p. 250).

9 Dans une logique du journaliste spécialisé à l'ère du numérique poussée à son paroxysme, nous pourrions aussi nous demander si l'élitisme qui entoure souvent la perception du grand public à l'endroit du journalisme français notamment ne serait pas aujourd'hui un peu diluée dans cet espace sans frontière que représente le web. Le journalisme spécialisé prendrait-il alors sa revanche sur le vieil adage caractéristique du journaliste généraliste considéré comme « Touche à tout, bon à rien »?

\section{NOTES}

1. Bonnafous S. (1999). «La médiatisation de la question immigrée : état des recherches ». In Études de Communication, $\mathrm{n}^{\circ}$ 22, p. 59-72.

2. Neveu E., Rieffel R., Ruellan D. (2002). « Journalistes spécialisés. Présentation, 10 ans après... ». In Réseaux, vol. 20, $\mathrm{n}^{\circ}$ 111, p. 9-17.

3. Charon J.-M. (1993). Cartes de Presse, Stock, Paris ; ou encore Charon J.-M., Papet J. (dir.) (2016). Le journalisme en questions. Nouvelles frontières des médias et du journalisme, Paris, L'Harmattan.

\section{AUTEURS}

\section{MARIE-ÈVE SAINT GEORGES}

Univ. Lille, ULR 4073 - GERiiCO, F-59000 Lille, France

marie-eve.saint-georges@univ-lille.fr 